\title{
O(s) Sistema(s) Primário(s) de Saúde Canadense sob uma perspectiva brasileira: discutindo os Atributos Starfield
}

\author{
The Canadian Primary Health Care Systems from a Brazilian \\ perspective: discussing Starfield's Attributes
}

José Ricardo de Mello Brandão (https://orcid.org/0000-0002-9131-3701) ${ }^{1}$

${ }^{1} \mathrm{MCI}$ The Doctor's Office at Mt. Pleasant. 245 Eglinton Av. East, M4P 3B7. Toronto ON Canadá. jrbranda.ops@gmail.com

\begin{abstract}
This paper reviews the Starfield pillars and the Canadian health system. An objective and subjective evaluation are applied to the system through the lenses of access, longitudinality, integrality, and coordination of care. System vulnerabilities, actions, and proposals that are underway to improve these aspects, both nationally and in the province of Ontario, are discussed. Worth highlighting is the opportunity to establish a national free drug system, and the several challenges to advance the agenda of reforms.
\end{abstract}

Key words Primary health care, Health system, Canada
Resumo Os pilares Starfield são revistos assim como o sistema de saúde canadense. Avaliação objetiva e subjetiva são aplicadas ao sistema sob as lentes do acesso, longitudinalidade, integralidade e coordenação de cuidados. Discutem-se as vulnerabilidades do sistema e as ações e propostas que estão em curso para tentar melhorar esses aspectos, tanto nacionalmente como na província de Ontário. Destaque para a oportunidade de se criar um sistema gratuito de medicamentos e os diversos desafios para avançar a agenda de reformas.

Palavras-chave Atenção primária à saúde, Sistema de saúde, Canadá 
Pode parecer pretensioso para um brasileiro manifestar-se a respeito do sistema de saúde canadense haja visto que publicação recente de avaliação de praticamente todos os países do mundo colocou o sistema canadense em um respeitável $17^{\circ}$ lugar, enquanto o Brasil amargava o $95^{\circ}$ posto ${ }^{1}$. Por outro lado, somos um povo curioso e queremos conhecer um pouco do que acontece em países em outro estágio de desenvolvimento (o que obviamente vai se refletir no seu sistema de saúde) e, quem sabe, vislumbrar soluções ou questões que nos ajudem a diminuir a nossa distância dos países ditos desenvolvidos.

A régua que procuraremos de alguma maneira utilizar, são os atributos ou pilares da Atenção Primária à Saude (APS) criados pela brilhante Profa ${ }^{\text {. }}$. Barbara Starfield (1932-2011). Eles seguem sendo conceitos importantes para se tentar dissecar aspectos da atenção à saúde. São eles, usando as traduções utilizadas no nosso Tratado de Medicina de Família (TMF)², a atenção ao primeiro contato/acesso (first contact care/gatekeeper), longitudinalidade (longitudinality and managed care, também referido como continuity of care), cuidado abrangente/integralidade (comprehensiveness and benefit packages no original, comprehensive care mais recentemente) e coordenação (coordination and the process of referral, coordination of care na sua versão encurtada). Suas definições e detalhamentos podem ser encontrados no livro seminal da autora ${ }^{3}$ e interessantes exemplos de suas traduções para a realidade brasileira, principalmente no contexto da Estratégia da Saúde da Família (ESF), podem ser apreciadas no capítulo correspondente do $\mathrm{TMF}^{2}$. Embora os 'iniciados' não tenham dificuldade para reconhecer do que a autora está falando vou me arriscar a simplificá-los aqui.

O primeiro deles, provavelmente o que abarca a maior gama de aspectos da APS, envolve todas as questões em relação ao acesso, sejam elas geográficas, disponibilidade do profissional, características socioculturais que influenciam esse acesso ou as tecnologias que o intermediam (ampliando a definição para a nossa realidade atual). Mas, vai além. Destaca a importância do profissional, preferencialmente um generalista, que fará a entrada do paciente no sistema.

$\mathrm{O}$ segundo refere-se à importância do paciente ser seguido por um mesmo profissional de saúde ou uma mesma equipe/local.

$\mathrm{O}$ terceiro pilar explora o aspecto holístico (ou não) do cuidado. As necessidades dos pacientes são potencialmente amplas e prover cuidados integrais (e ser capaz de bancá-los no sistema) é sempre um grande desafio.
O quarto refere-se à capacidade do sistema e dos profissionais envolvidos em efetivamente se comunicarem e manterem a racionalidade no cuidado ao paciente. Sua manutenção evitaria interrupções no cuidado ou duplicações das intervenções, ambas com potencial prejuízo ao paciente. O provável 'cálice sagrado' do conceito seria o prontuário único (supondo-se que ele seria lido pelos profissionais envolvidos no cuidado ao paciente).

A avaliação desses atributos em determinado sistema é tema de uma extensa bibliografia incluindo instrumentos criados com a colaboração da autora, como o Primary Care Assessment Tool (PCAT), descrito no nosso $\mathrm{TMF}^{2}$ e bastante utilizado no Brasil. Mas, optamos por utilizar outros indicadores objetivos, relacionando-os com os atributos, assim como opiniões de especialistas e, eventualmente, do próprio autor, de maneira a trazer um recorte o mais atualizado possível do sistema canadense. Naturalmente, as últimas incluirão sua dose de subjetividade.

E já que o sistema canadense será o objeto do ensaio, vamos também revê-lo brevemente. Excelente descrição foi publicada em 2017 no Lancet $^{4}$ e será fonte do nosso resumo abaixo. O chamado Medicare (não confundir com o Medicare americano que se limita à cobertura da população acima dos 65 anos e parte da população mais jovem com incapacidades) corresponde ao conjunto de sistemas provinciais que teve sua origem na província de Saskatchewan em 1947 e foi replicado em outras províncias nas décadas seguintes. A "colcha de retalhos" foi harmonizada com uma lei federal em 1984 (Canadian Health Act). Há inclusive autores que o definem como um "seguro de saúde com pagador único" (single-payer) ao invés de um verdadeiro sistema ${ }^{5}$, para não mencionar que a autonomia das províncias produz, em realidade, múltiplos "sistemas".

A história da criação do sistema canadense nos permite entender os limites da atualidade já que as características por detrás da proposta original na década de 40 (curativa, baseada em hospitais, focada na figura do médico) é completamente diversa das principais necessidades da atenção à saúde desse século (prevenção, cuidados ambulatoriais e necessidade de intervenção multiprofissional).

No seu atual funcionamento, o sistema canadense pode ser visto como atuante em três camadas. A primeira, virtualmente toda coberta pelo sistema público, incluiu o atendimento integral em hospitais, consultas médicas e exames diagnósticos. A segunda, com cobertura apenas par- 
cial e de maneira mais excepcional, envolve medicamentos prescritos (em Ontário, por exemplo, para maiores de 65 anos e jovens abaixo de 25 , esses últimos apenas se não tiverem cobertura privada, dependentes do sistema de bem-estar ou com gastos "catastróficos em saúde" - mais de 4\% da sua renda), cuidados domiciliares (home care), casas de repouso para idosos (long term care, fundamental aspecto do cuidado em um país onde morar com os filhos no final da vida não é o mais habitual) e saúde mental. A terceira camada, virtualmente toda paga com dinheiro próprio (out of pocket) ou de seguros privados, envolve a saúde oral, saúde ocular, medicina complementar, incluindo a fisioterapia ambulatorial.

Médicos de família (MF) formam a base do sistema e correspondem grosso modo à metade dos profissionais médicos do país (122 médicos de família/100.000 hab vs 119 especialistas/100.000 hab) $)^{5}$. Segundo o mesmo relatório, o crescimento do total de médicos desde 2014 foi o dobro do crescimento populacional, o que tende a favorecer o acesso. Recebem majoritariamente pagamentos por consulta/procedimento (fee for service) em comparação a pagamentos alternativos (definidos como salários, capitação, pagamento por hora ou sessões, contratos): $72,6 \%$ vs 27,4\% em 2018 (diferença voltando a aumentar discretamente nos últimos cinco anos mostrando uma estabilização na tendência que vinha desde a década de 90 de aumento dos pagamentos alternativos) ${ }^{6}$.

Os MF trabalham geralmente sem suporte de uma equipe multiprofissional. Na tentativa de mitigar essa realidade, formou-se em Ontario, entre 2005 e 2012, 184 equipes de Saúde da Família (Family Health Teams - FHT), sobretudo em ambientes universitários e locais de população de risco, incluindo o norte da província, comunidades rurais e servindo populações vulneráveis nos grandes centros urbanos 7 . Desde 2015, no entanto, não há autorização para formação de novas equipes. Uma nova tentativa do atual governo está propondo uma reforma substancial do sistema, sempre na linha do trabalho multiprofissional, com a criação das equipes de saúde de Ontario $(\text { Ontario Health Teams }-\mathrm{OHT})^{8}$. Voltaremos à essa proposta mais à frente.

Voltando à avalição do sistema, utilizaremos dados da última rodada da pesquisa de política de saúde realizada pelo Commonwealth Fund em $2016^{9}$, com publicações comparando os dados de onze países de alto nível de renda: EUA, Reino Unido, Canadá, Alemanha, Austrália, Japão, Suécia, França, Holanda, Suíça e Dinamarca ${ }^{10,11}$ tentando relacioná-los com os atributos da conclamada autora.

Em termos de acesso, temos que $43 \%$ dos canadenses ( $8^{\circ}$ lugar no grupo) conseguem marcar uma consulta para o mesmo dia ou para o dia seguinte com o seu MF, para uma média de $57 \%$; $39 \%$ (pior desempenho entre esses países) esperam ao menos dois meses para conseguir ver um especialista (para uma média de 13\%); 34\% dos canadenses consideram fácil ou relativamente fácil atendimento after-hours - noites, finais de semanas e feriados - sem recorrer à emergência (penúltimo lugar) para uma média de $43 \%$; em saúde mental, 59\% conseguem ajuda profissional quando necessitam, acima da média de 54\%; no entanto, $41 \%$ dos canadenses estiveram em uma emergência nos últimos 2 anos (último lugar), para uma média de $27 \%$; e quando vão, $29 \%$ esperam $4 \mathrm{~h}$ ou mais para o atendimento, pior desempenho para uma média de $11 \%$; o quadro se repete para espera de quatro meses ou mais para uma cirurgia eletiva: novamente última colocação com $18 \%$ das pessoas, para uma média dos países do fundo de $9 \%$. Isso mostra um claro problema de acesso, embora alguns desses indicadores referem-se à atenção secundária ou terciária. No entanto, pode-se questionar alguns desses indicadores (por exemplo, consulta no mesmo dia ou no próximo) já que o acesso a essa consulta não implica necessariamente em um cuidado adequado com melhora da qualidade de vida de quem busca o atendimento ${ }^{5}$. Além disso, embora estudando apenas pessoas que tinham um MF na província de Ontario, uma avaliação mais ampla do acesso mostrou que essa população tem em sua maioria impressões positivas desse acesso ${ }^{12}$.

Mas, na linha do acesso à saúde em geral, a grande barreira no Canadá segue sendo a inexistência de um plano nacional de Pharmacare, ou seja, sistema de medicamentos gratuitos à população. O Canadá é o único país do mundo com um sistema de saúde universal que não tem essa cobertura ${ }^{13}$. A importância da incorporação de medicamentos gratuitos ao sistema é amplamente apoiada pelos especialistas ${ }^{4,5,11,13-16}$ inclusive com a demonstração das vantagens econômicas. O Partido Liberal do Canadá, vencedor minoritário das eleições de outubro último, tem em sua plataforma eleitoral proposta e orçamento destinados a apoiar as províncias para ampliação do acesso a fármacos. O provável partido que deve apoiá-lo para permitir o governo minoritário, o Novo Partido Democrático (New Democratic Party - NDP), tem proposta e orçamento ainda mais robustos no apoio à medida. 
Ademais, entre os principais partidos, apenas o partido conservador não se comprometia à criação do Pharmacare, comprometendo-se apenas a aumentar o acesso aos medicamentos para patologias $\operatorname{raras}^{17}$. Mas, dada a necessidade de se negociar com as treze províncias e territórios, algumas governadas pelo derrotado partido conservador, prevê-se um árduo processo à frente ${ }^{18}$. $\mathrm{E}$ a questão não se limita ao momento político atual. Múltiplos outros aspectos precisam ser negociados como, por exemplo, o fato de que com a criação de um fundo federal para compras de medicamentos (beneficiando-se de economia de escala), as províncias que hoje compram seus medicamentos teriam que transferir/retornar dinheiro para o governo central ${ }^{5}$. E o Quebec é um caso particular. A província tem hoje um sistema onde o cidadão que não tem seguro privado cobrindo medicamentos é obrigado a pagar por sistema semelhante governamental (garantindo sua cobertura medicamentosa), com vantagens e desvantagens reconhecidas ${ }^{19}$. Seu modelo poderia ser seguido no restante do país ou eventualmente o Quebec conseguiria manter-se à parte, dada algumas características de autonomia que a província possui (hoje o seu sistema de bancos de sangue, por exemplo, é independente $)^{20}$. Se as possibilidades anteriores não ocorrerem, a província teria que fazer um grande ajuste.

Mas, voltemos à avaliação dos atributos. No quesito longitudinalidade (e também qualidade do cuidado), $85 \%$ dos canadenses têm um "médico habitual" (usual doctor), média dos países do fundo. Fazem um elevado número de consultas/ ano (7.6 vs 5.8 de média), tem uma melhor percepção do seu médico do que a média (médico detém informações importantes, gasta tempo suficiente na consulta e explica o que está acontecendo de maneira acessível) e têm seus medicamentos revistos mais frequentemente $(77 \%$ vs $68 \%)$. Surge, portanto, como um dos pontos altos do sistema canadense, posição compartilhada por outros autores ${ }^{21}$.

Em relação à abrangência de cuidados, temos menos dados objetivos. A baixa prevalência de equipes multiprofissionais, já comentada, aponta para a principal barreira para um cuidado mais holístico da população canadense. Adicione-se a isso a virtual ausência de saúde oral gratuita $(28 \%$ dos canadenses relatam deixar de ir ao dentista devido ao custo vs $20 \%$ da média internacional), aumentando o déficit de integralidade. Acrescente-se a não cobertura de outros profissionais de saúde (fisioterapeutas, psicólogos, fonoaudiólogos, por exemplo) descrita an- teriormente e as limitações estão expostas. Essas coberturas são apontadas como urgenciais ${ }^{4}$ para a melhora do sistema. Em defesa da abrangência de cuidados no sistema canadense, vale ressaltar a excelente formação clínica do médico de família o que lhe permite um cuidado diferenciado da sua população. Destaque-se também a homogeneidade dessa formação em um território vasto e com realidades culturais razoavelmente diversas. No Brasil, o caráter multiprofissional inerente da ESF, incluindo cobertura em saúde bucal se aproximando de $50 \%$ da população ${ }^{22}$, destaca-se no quesito abrangência de cuidados dentro do Sistema Único de Saúde (SUS). Curiosamente, na província de Ontário, a tentativa de bancar a formação de FHT mostrou-se cara e com resultados aquém dos esperados ${ }^{5,23}$ mostrando a complexidade na proposição de mudanças no sistema.

Dados objetivos para se avaliar coordenação de cuidados são mais difíceis de serem encontrados. Apesar do crescimento na adoção de prontuários médicos eletrônicos ${ }^{24}$, compatibilizá-los é sempre um enorme desafio. Nenhuma província chegou à criação de um prontuário único que pudesse ser utilizado em todos os níveis, embora Alberta tenha avançado nesse sentido ${ }^{5}$. Ontário também tem diversos projetos, capitaneados por eHealth Ontario, com destaque para o Connecting Ontario ClinicalViewer, um sistema que permite ao profissional médico acesso a relatórios de consultas e atendimentos comunitários, exames laboratoriais e de imagem dos principais hospitais da província, assim como informação dos medicamentos fornecidos pelas farmácias para qualquer paciente inscrito no sistema provincial ${ }^{25}$.

Mas a integração entre os diversos cuidadores e uma transição eficiente entre eles requer mais do que um prontuário único. Ontário está se lançando em um ambicioso projeto, os OHT, que procura integrar potencialmente todos os atores envolvidos com o cuidado em saúde da população, com catorze áreas incluídas no documento inicial, indo da atenção primária à reabilitação e cuidados complexos, passando por diagnósticos, serviços de suporte comunitário e cuidados paliativos, entre outros ${ }^{7}$. Nos últimos meses o Ministério da Saúde $(\mathrm{MoH}$ - aqui as províncias usam o termo ministério que na verdade equivale às nossas secretarias) convidou provedores a formarem grupos e pleitear a sua aprovação. Das mais de 150 propostas apresentadas, 31 foram aprovadas inicialmente (com outros 43 grupos encorajados a melhor desenvolverem seus projetos) e tiveram que apresentar uma proposta mais completa $^{26}$, entregues em outubro último. Faço 
parte de um destes 31 grupos (North Toronto) que decidiu priorizar o atendimento de idosos num primeiro momento, a ser seguido por cuidados em saúde mental e crianças/joven ${ }^{27}$. Responsabilizando-se por uma população de cerca de 180.000 mil pessoas, a proposta é detalhada e inclui a integração dos principais sistemas de informações usados pelos parceiros. A expectativa é que sete a dez grupos serão escolhidos, ainda no final de 2019, para formarem oficialmente os primeiros $\mathrm{OHT}^{28}$.

Apesar de uma grande receptividade por parte dos provedores, a proposta enfrenta críticas, sobretudo em relação às inúmeras dúvidas em relação à sua governança ${ }^{29}$.

Perpassando as questões apresentadas, gostaria de comentar mais dois importantes aspectos da realidade de saúde canadense. Um de natureza mais aguda e crítica, outro mais complexo, crônico e de alto simbolismo. O primeiro refere-se ao crescente número de idosos ocupando leitos hospitalares enquanto aguardam vaga em uma residência para idosos (nursing homes). São pessoas consideradas incapazes de retornarem a seus lares, após uma hospitalização, mesmo frente a um apoio (às vezes irregularmente oferecido) em casa. Seus números têm batido recordes na província de Ontário ${ }^{30}$ e levam, naturalmente, a um consequente aumento de pacientes esperando para serem internados, em macas, nos prontos-socorros. A chamada 'medicina de corredor' (hallway medicine), familiar a nós brasileiros, choca-nos ao ser vista em um país como o Canadá, a ponto de levar a minha geração de médicos a longos debates quando presenciamos essa realidade em cenas do premiado filme canadense de 1986, dirigido por Denys Arcand, "O declínio do Império americano". Mais de trinta anos depois, o quadro ainda é comum em grandes cidades como Toronto e Montreal. A evidente necessidade de se construir mais residências para idosos tem encontrado uma resposta mitigada do governo ${ }^{31}$.

$\mathrm{O}$ segundo fenômeno que se tem mostrado claramente necessitando de maiores e mais adequados investimentos é o da iniquidade existente entre as condições de vida e saúde das populações indígenas em relação à população não indígena $^{32}$. Está claro que as intervenções necessárias ultrapassam o limite da saúde e envolvem mudanças nos determinantes sociais da saúde para mudarmos um quadro inaceitável ${ }^{4} \mathrm{em}$ um país rico como o Canadá.

Temos então, em conclusão, um sistema de saúde que se destaca por uma atenção primária fundada primordialmente na atuação de médicos de família que garantem um excelente nível de longitudinalidade. Sua distribuição pelo país permite um acesso bastante razoável à população, embora certa desigualdade persista. Acesso a consultas em um curto prazo de tempo segue problemático. Sólida formação permite aos médicos uma ampla abrangência de cuidados na área clínica, mas o pequeno número de equipes multiprofissionais limita as intervenções ampliadas em saúde. Continuidade de cuidados é notoriamente deficiente, mas tem-se concentrado esforços para o seu aprimoramento.

Em um país que pelo quarto ano seguido liderou a lista dos países com a melhor qualidade de vida do mundo ${ }^{33}$, particularmente quando sabemos que seu sistema público de saúde contribuiu para esse destaque ${ }^{34}$, é interessante notar como uma lente de aumento permite vislumbrar que ainda há muito a ser feito. Mas, curiosamente, o sistema pode sofrer do velho ditado de que o "bom é inimigo do ótimo", como argumentam com perspicácia autores já aqui citados ${ }^{5}$. Ou seja, em um sistema bastante funcional, a pressão dos cidadãos para que mudanças ocorram é menos presente. E a complexidade do sistema federativo canadense impõe necessidades de sofisticadas negociações, inibindo de certa maneira o avanço da agenda de mudanças. Embora, na minha opinião, a maturação de certas propostas, descritas no corpo do artigo, mostram como governantes que creem num sistema universal de saúde e estão comprometidos com a melhoria desse sistema podem fazer sua contribuição. Permitindo o aperfeiçoamento de um sistema de saúde que segue sendo um modelo mundial. 


\section{Referências}

1. GBD 2015 Healthcare Access and Quality Collaborators. Healthcare Access and Quality Index based on mortality from causes amenable to personal health care in 195 countries and territories, 1990-2015: a novel analysis from the Global Burden of Disease Study 2015. Lancet 2017; 390(10091): 231-266.

2. Gusso G, Machado LBM. Atenção Primária à Saúde. In: Gusso G, Lopes JMC, Dias LC. Tratado de Medicina de Família e Comunidade. Princípios, Formação e Prática. 2a ed. Porto Alegre: ArtMed; 2019. p. 28-36.

3. Starfield B. Primary Care: Concept, Evaluation, And Policy. New York: Oxford University Press; 1992.

4. Martin D, Miller AP, Quesnel-Vallée A, Caron NR, Vissandjée B, Marchildon GP. Canada's universal health-care system: achieving its potential. Lancet 2018; 391(10131):1718-1735.

5. Ivers N, Brown AD, Detsky AS. Lessons From the Canadian Experience With Single-Payer Health Insurance. Just Comfortable Enough With the Status Quo. JAMA Intern Med 2018; 178(9):1250-1255.

6. Canadian Institute for Health Information. Ottawa: Physicians in Canada [página na Internet]. 1996-2019 [acessado 2019 Out 27]. Disponível em: https://www. cihi.ca/en/physicians-in-canada

7. Ontario Ministry of Health/Ministry of Long-Term Care. Toronto: Family Health Teams [página na Internet]. 2016 [acessado 2019 Out 27]. Disponível em: http://health.gov.on.ca/en/pro/programs/fht/

8. Ontario Ministry of Health/Ministry of Long-Term Care. Toronto: Become an Ontario Health Team [página na Internet]. 2019 [acessado 2019 Out 27]. Disponível em: http://health.gov.on.ca/en/pro/programs/connectedcare/oht/

9. Organisation for Economic Co-operation and Development (OECD). Paris: OECD Health Statistics 2019 [página na Internet]. 2019 [acessado 2019 Out 27]. Disponível em: http://www.oecd.org/els/health-systems/health-data.htm

10. Schneider EC, Sarnak DO, Squires D, Shah A, Doty MM. Mirror, Mirror 2017: International Comparison Reflects Flaws and Opportunities for Better U.S. Health Care [página na Internet]. New York: The Commonwealth Fund; 2017 [acessado 2019 Out 27]. Disponível em: https://www.commonwealthfund.org/publicatio ns/fund-reports/2017/jul/mirror-mirror-2017-international-comparison-reflects-flaws-and

11. Canadian Institute for Health Information. How Canada Compares Results From The Commonwealth Fund's 2016 International Health Policy Survey of Adults in 11 Countries [página na Internet]. 2017 [acessado 2019 Out 27]. Disponível em: https://www.cihi.ca/sites/default/files/document/text-alternative-version-2016cmwf-en-web.pdf

12. Premji K, Ryan BL, Hogg WE, Wodchis WP. Patients' perceptions of access to primary care - Analysis of the QUALICOPC Patient Experiences Survey. Can Fam Physician 2018; 64(3):212-220.

13. The Lancet. Canada Needs Universal Pharmacare. Lancet 2019; 394(10207):1388.

14. Morgan SG, Law M, Daw JR, Abraham L, Martin D. Estimated cost of universal public coverage of prescription drugs in Canada. CMAJ 2015; 187(7):491497.
15. Martin D. Better now: six big ideas to improve health care for all Canadians. Toronto: Allen Lane; 2017.

16. Bell R, Superina S, Raza D. Comparing Health Systems: Lessons for Ontario [página na Internet]. Drbobbell; 2019 [acessado 2019 Out 27]. Disponível em: https:// drbobbell.com/comparing-health-systems-lessons-for-ontario/

17. The Globe and Mail. Federal election 2019: The definitive guide to the issues and party platforms [página na Internet]. The Globe and Mail; 2019 [acessado 2019 Out 27]. Disponível em: https://www.theglobeandmail.com/politics/article-party-platforms-guide-canadian-federal-election-2019/

18. Cohn MR. Doug Ford will kill the pharmacare ambitions of Justin Trudeau and Jagmeet Singh, just like he gutted OHIP+ [página na Internet]. The Star; 2019 [acessado 2019 Out 27]. Disponível em: https://www. thestar.com/politics/political-opinion/2019/09/30/ doug-ford-will-kill-the-pharmacare-ambitions-ofjustin-trudeau-and-jagmeet-singh-just-like-he-gutted-ohip.html

19. Rolfe K. Could Quebec's public drug plan work for national pharmacare? [página na Internet]. Benefits Canada; 2019 [acessado 2019 Out 27]. Disponível em: https://www.benefitscanada.com/news/quebec-public-private-drug-plan-a-viable-solution-to-nationalpharmacare-report- 133472

20. Picard A. National pharmacare is possible - but it won't come easy [página na Internet]. The Globe and Mail; 2018 [acessado 2019 Out 27]. Disponível em: https:// www.theglobeandmail.com/opinion/article-nationalpharmacare-is-possible-but-it-wont-come-easy/

21. Damji AN, Martin D, Lermen N, Pinto LF, Trindade TG, Prado JC. Trust as the foundation: thoughts on the Starfield principles in Canada and Brazil. CMAJ 2018; 64(11):811-815.

22. Índice de Desempenho do Sistema Único de Saúde (Idsus). Brasília: Cobertura estimada da população residente pelas equipes de saúde bucal da atenção básica [página na Internet]. 2011 [acessado 2019 Out 27]. Disponível em: http://idsus.saude.gov.br/ficha2s.html

23. Grant K. Ontario's curious shift away from family health teams [página na Internet]. The Globe and Mail; 2015 [acessado 2019 Out 27]. Disponível em: https:// www.theglobeandmail.com/life/health-and-fitness/ health/ontarios-curious-shift-away-from-family-health-teams/article22989363/

24. Ubelacker S. More Canadian doctors embracing electronic medical records [página na Internet]. The Canadian Press, CTV News; 2016 [acessado 2019 Out 27]. Disponível em: https://www.ctvnews.ca/health/ more-canadian-doctors-embracing-electronic-medical-records-1.2755721

25. Ontario Health. ConnectingOntario ClinicalViewer [página na Internet]. 2008-2019 [acessado 2019 Out 27]. Disponível em: https://www.ehealthontario.on. $\mathrm{ca} / \mathrm{en} /$ for-healthcare-professionals/connectingontario

26. Ontario Ministry of Health/Ministry of Long-Term Care. Toronto: Become an Ontario Health Team. Healthcare professionals. Ontario Health Teams: List of Teams Invited to Full Application and In Development for Public Posting [página na Internet]. 2019 [acessado 2019 Out 27]. Disponível em: http://health.gov.on.ca/ en/pro/programs/connectedcare/oht/teams.aspx 
27. North Toronto Ontario Health Team. Toronto: Our application: next steps [página na Internet]. 2019 [acessado 2019 Out 27]. Disponível em: http://northtorontooht.ca/\#nextsteps

28. Grant K. More than 150 groups apply to become Ontario Health Teams as competition for care intensifies [página na Internet]. The Globe and Mail; 2019 [acessado 2019 Out 27]. Disponível em: https://www.theglobeandmail.com/canada/article-more-than-150-groupsapply-to-become-ontario-health-teams-as/

29. LaFleche G, Frketich J. How Ontario's new regional health teams will operate is anyone's guess in 'low-rules environment' [página na Internet]. Hamilton Spectator and St. Catharines Standard; 2019 [acessado 2019 Out 27]. Disponível em: https://www.thespec.com/ news-story/9510864-how-ontario-s-new-regionalhealth-teams-will-operate-is-anyone-s-guess-in-lowrules-environment-/

30. Boyle T. Number of seniors waiting to move into long-term care homes in Ontario hits record high [Internet]. The Toronto Star; 2019 [acessado 2019 Out 27]. Disponível em: https://www.thestar.com/news/ gta/2019/09/16/more-seniors-than-ever-waiting-forlong-term-care-beds-in-ontario.html (Requer asinatura).

31. Crawley M. Nursing home group wants more beds, bigger construction subsidy [Internet]. CBC News; 2017 [acessado 2019 Out 27]. Disponível em: https://www.cbc.ca/news/canada/toronto/ontario-long-term-care-beds-association-nursing-home1.4371629

32. Adelson A. The Embodiment of Inequity: Health Disparities in Aboriginal Canada. Can J Public Health Mar 2005; 96(Supl. 2):S45-S61.

33. US News \& World Report. Quality of Life Rankings [página na Internet]. 2020 [acessado $2020 \mathrm{Fev}$ 17]. Disponível em: https://www.usnews.com/news/best -countries/quality-of-life-rankings

34. Shepert E. Study ranks Canada \#1 in the world for quality of life [página na Internet]. Vancouver Courier; 2019 [acessado 2020 Fev 17]. Disponível em: https:// www.vancourier.com/news/study-ranks-canada-1in-the-world-for-quality-of-life-1.23609916 
